\title{
Calcium granules transport across the endoderm of the pigeon
}

\author{
Juan Song ${ }^{1, a}$, Hua Tong ${ }^{2, b}$, Haixia Cheng ${ }^{2, c}$ \\ ${ }^{1}$ Analytical and Testing Center, Hubei University of Technology, Wuhan 430068, People's Republic \\ of China \\ ${ }^{2}$ College of Chemistry and Molecular Sciences, Wuhan University, Wuhan 430072, People's \\ Republic of China \\ aemail:songjuan09@126.com, bemail:huatong09@126.com, cemail:308932915@qq.com
}

Keywords: pigeon; calcium; transport; endoderm

\begin{abstract}
Calcium granules are known to form in the yolk sacs of fertile pigeon eggs at late stages of incubation. The appearance regularity, mineralization and demineralization position and tracking of calcium granules with the incubation process are investigated. Polarized light microscopy was used to observe the generation and distribution of calcium carbonate crystals in the yolk sac. The results of Polarized light microscopy observation show that the calcium granules density in endoderm increases with the change of incubation time, and the volume of calcium granules also increases constantly. SEM observes that calcium granules in endoderm are wrapped in domain structure. EDS shows that the content of calcium in endoderm increases constantly, which indicates that the absorption rate of endoderm on calcium increases. Calcium-rich particles, scattered zones of minute calcium-rich particles and calcium granules in cells of endoderm are found in endoderm, which shows that endoderm has degradation and absorption function on calcium-rich particles. These observations confirm the calcium absorption rate of endoderm gradually increases as the incubation time changes, which achieves maximum around middle stage of incubation, the calcium absorption rate declines slightly after incubation. The endoderm has the similar endocytosis to spherical calcium granules.
\end{abstract}

\section{Introduction}

There have been a large number of studies about the metabolism of various nutrient substances in yolk sac during the incubation, including sugar, amino acid, water, protein, fat, various minerals and vitamins, etc [1-6]. Calcium is one of the elements which are most abundant in poultry, and also the largest influencing element to the embryonic development during the incubation. The study of embryo in early incubation verifies that the sharp decrease of calcium content in yolk sac is mainly to meet the early embryonic development, in later incubation, the calcium in albumen and yolk sac transports to the embryo, and stores a lot of calcium in the yolk sac to meet the adult development after hatching [7]. The research about the transport process of calcium in the embryo is generally believed that the calcium transships into the embryos is mainly through the endoderm and chorioallantoic membrane [8-10]. As a kind of complex membrane of embryo, the endoderm is mainly responsible for transporting the nutrients needed by tissue development from the yolk sac into the embryo [11].Shinji Komazaki [12] chooses the chicken oosperm which is incubated nine days as the research object, the calcium is transported from the yolk sac into embryonic circulation in this stage, and the result confirms that the endoderm has the function of the calcium absorption and transport, and it shows endocytosis to the yolk sac material which is containing calcium.

Our previous research investigated the development process of pigeon's embryo, the most significant change of yolk substance found in hatching process is transform from a disordered state 
to an ordered state. Calcium carbonate crystals largely appears in yolk sac in the middle and later periods of the development of pigeon's embryo, it increases gradually along with the developmental process, and gradually disappears after the embryos turns into pigeons (out of shell)[13]. The rule of calcium activity which is connected with the process of embryonic development, its important biological significance is that it may be the most effective form that the embryo uses the calcium. This experiment was conducted to bring the crystalline substance into the category of biological mineralization from the perspective of biological crystalline substance, and inspect the appearance, growth and absorption rule on endoderm of crystalline substance of calcium at different stages of incubation, what will have very important significance to understand the transport process of calcium and its biological function.

\section{Materials and methods}

\section{Materials and instruments}

The pigeon eggs used in our experiments were obtained from the pigeon farm of Central China Agriculture University. They were incubated in batches in a tabletop incubator (with automatic turning) maintained at $38.2 \pm 0.1{ }^{\circ} \mathrm{C}$ with relative air humidity of $60 \pm 5 \%$. Eggs were candled after 5 days; dead and infertile eggs were discarded. Polarized light microscopy (PLM; Sunny XP, China), scanning electron microscopy (SEM; QUANTA FEG450, FEI, the Netherlands), energy dispersive analysis (EDX; Oxford, UK) were employed in this study.

\section{Experimental process}

\section{Granule collection from endoderm and PLM analysis}

Eggshells were carefully broken, the yolk sac separated from the egg white and embryo. The yolk sac endoderm of fertilized eggs incubated for $0,8,13$, or 18 days were subjected to PLM analysis. In order to maintain the native sample in the in vivo state our methods were free from any biochemical and histochemical processes. All the samples were prepared on glass slides for direct observation. Then the sample was put under PLM for observation. The PLM image was taken by a digital camera.

\section{SEM morphology observation and EDS elemental analysis.}

Endoderm was moved from the eggs attached to the vitelline membrane and transferred to Pannett Compton Saline [14]. Endoderm was fixed in 2.5\% glutaraldehyde solution for a further period of 12 hours. After fixation the endoderm was washed overnight in $0.2 \mathrm{M}$ sodium cacodylate buffer, postfixed in 1\% cacodylate buffered osmium tetroxide and dehydrated in ethanol. Specimens for SEM were passed from $100 \%$ ethanol to $100 \%$ acetone and critical point dried by replacing acetone with critical point dryer. Dried specimens were sputtered with a 20-nm gold film for morphology analysis by SEM using an ion sputtering apparatus. Micro-element identification was then carried out by EDS.

\section{Result and Discussion}

Spherical granules appear in the yolk sac endoderm at different developmental stages 
The endoderm was collected for PLM to observe the morphology and developmental process of spherical granules within yolk sac. The spherical granules appear on endoderm of yolk sac become less to more, and small to large (Fig1). The yolk sac is like a developing nutrition bag for a pigeon, and the nutrients attach on the inner membrane of the yolk sac before being distributed uniformly for the blood vessels to absorb [3]. The endoderm is in direct contact with the yolk sac and plays a role in the enrichment, decomposition, absorption, and transportation of nutrients [4]. Based on the fact that along with the incubation spherical granules appeared, developed and disappeared at the end of incubation, we can make a hypothesis: spherical granules as orderly state participate in embryonic development.
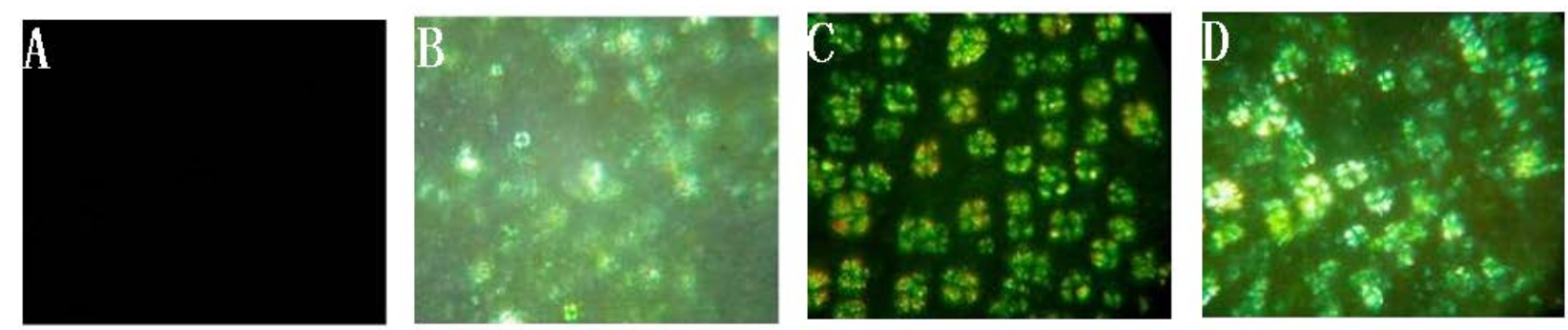

Figure 1 PLM images of yolk sac endoderm at different incubation times.

A-DMorphology at $0,5,13$, and 16 days of incubation.

\section{The change of content of calcium element on yolk sac endoderm}

In order to further clarify whether the transport process of calcium ion is related to the ordering of yolk substance, we use the SEM-EDS to observe the distribution and content change of calcium on endoderm in the incubation process of pigeon. From the SEM figure, we can see that the endoderm forms the obvious wrinkle in middle and later periods of incubation relative to the early period, it is consistent with the observation of PLM. The table1 lists the content change of various elements of pigeons' endoderm in different periods, and we can find the absorption rate of pigeons' endoderm to mineral elements in different periods (Fig2). During 4-12 days, the mineral elements on the endoderm have an obvious rising trend, e.g. $\mathrm{K}, \mathrm{Na}, \mathrm{Mg}$, $\mathrm{Ca}$, they have the highest content around the 12th day, then decline, and the element content is essentially balanced in the 15th and 16th days. The content change of mineral elements on endoderm is basically consistent with the changing tendency of conductivity in early development [13], but that shows a certain hysteresis. The calcium absorption rate of endoderm gradually increases as the incubation time changes, which achieves maximum around the 12th day, the calcium absorption rate declines slightly after incubation, but higher than in early development stage(0-8 days). 

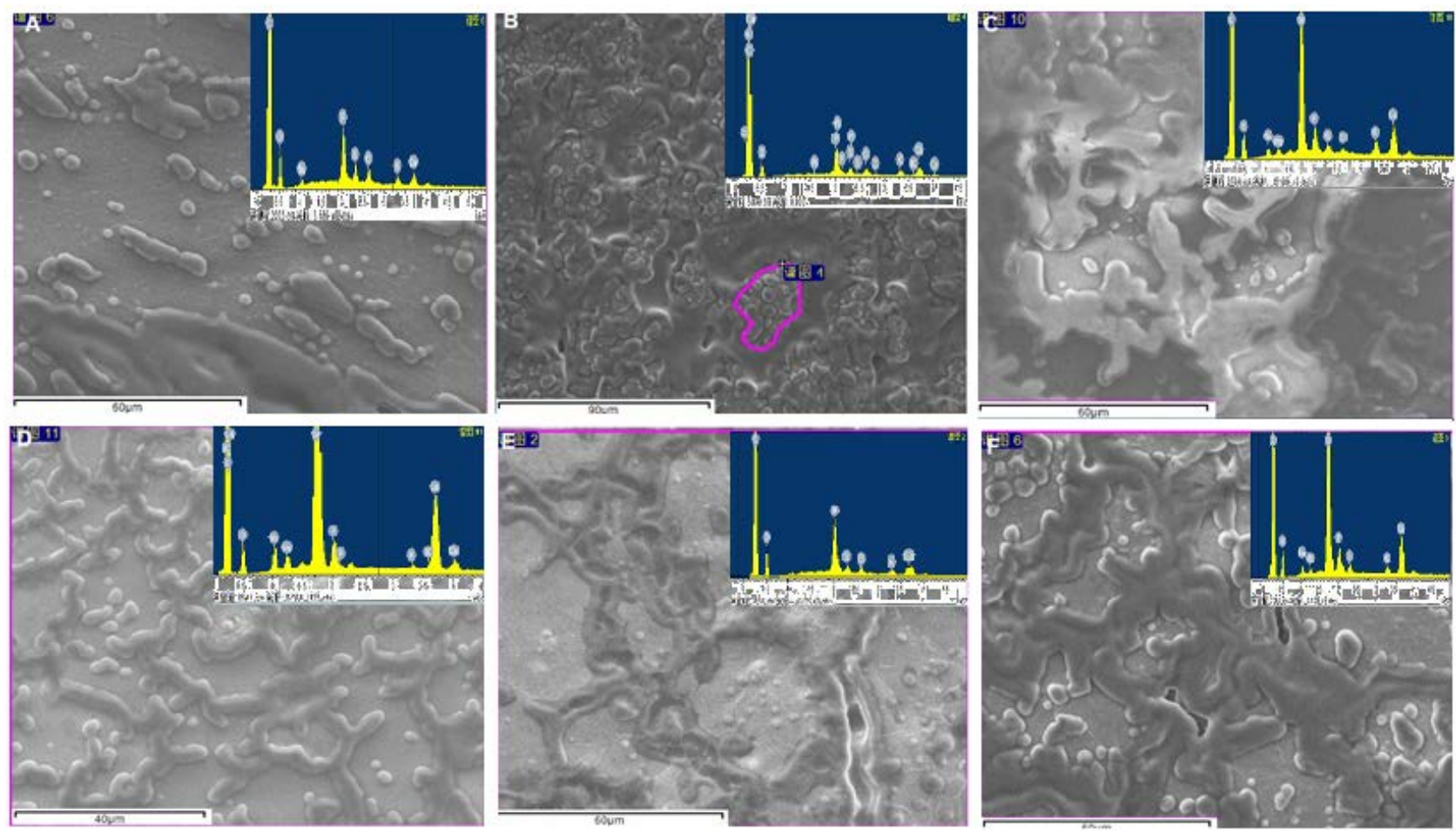

Figure 2 SEM and EDS images of yolk sac endoderm at different incubation times.

A、B、C、D、E、F: Morphology at 4, 8, 10, 13, 15and 16 days of incubation.

\section{Calcium granules transport process on endoderm of yolk sac}

In order to further understand the transport law of calcium granules on endoderm of yolk sac, we use the SEM-EDS to observe the spherical granules with higher calcium content on endoderm. Under the SEM, the size of spherical granules is ranging from tens of nanometers to hundreds of micrometers, and the granules can be distinguished from the membrane structure of surrounding endoderm. The result of EDS analysis shows that the calcium content of these granules is significantly higher than the surrounding tissue, the calcium content of spherical granules on pigeons' endoderm in the 13th hatching day can reach $1.73 \%$ and $1.23 \%$ (Fig3 ), the calcium content is $0.71 \%$ significantly higher than the surrounding tissue. Due to the size, morphology and granule size distribution of such spherical granule conform to the spherical granules observed by PLM, we judge these granules as calcium granules.

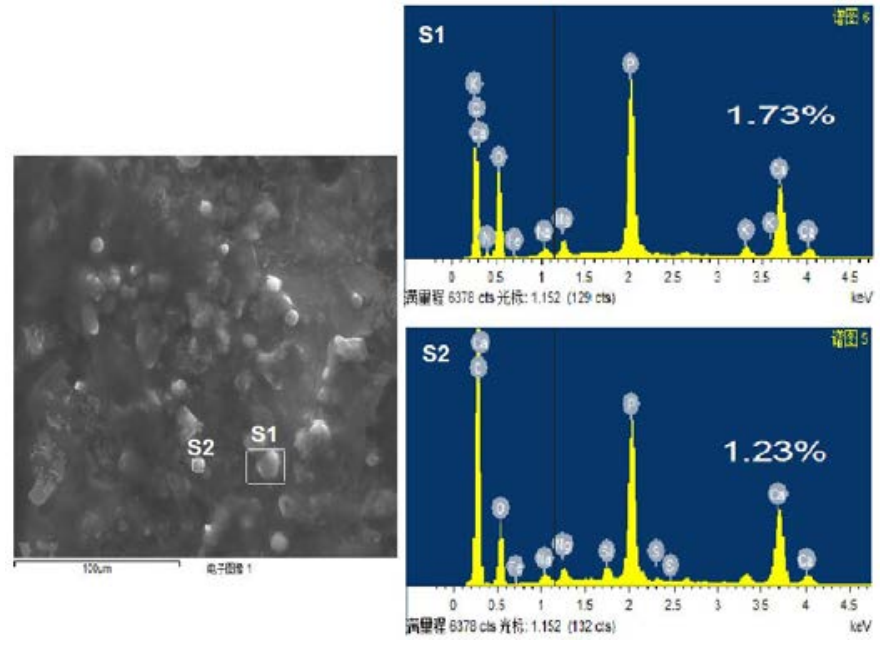

Figure 3 SEM and EDS images of yolk sac endoderm at in the 13th hatching day 
Previous study confirmed the calcium transport process of chickens' endoderm can be accumulated and coated through the calcium electron dense granules, and then spitted out of the cell through exocytosis[12]. By observing the distribution of calcium granule on pigeons' endoderm, we find the endoderm has the similar endocytosis to spherical calcium granules (Fig4). Early research affirms that in-layer of splanchnic wall of yolk sac can secrete enzymes to act on yolk substance, and then transport these substance into the embryo through the yolk blood vessels, but the transport process of yolk substance is not clear, through the experiment at this stage, we can learn that with the process of embryonic development, the wrinkles on wall of endoderm of yolk sac gradually increase, become dense, and coat the blood vessels, the calcium granules appear on endoderm of yolk sac become less to more, small to large, and fill with the whole endoderm and wrinkle, through the role of the enzyme, the calcium granules become pieces, and reach to the substructure which can be swallowed by endoderm, it again confirms that the transport process of calcium ion in yolk must go through the ordering process.
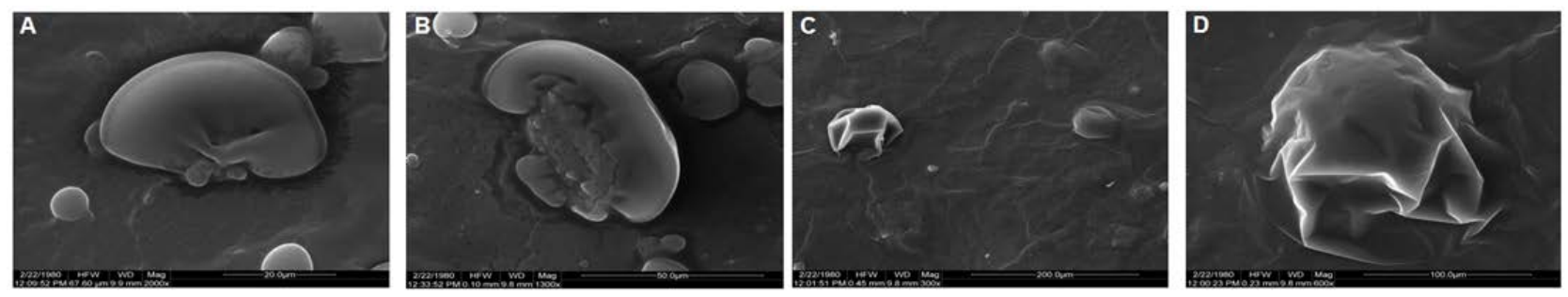

Figure 4 SEM image of the yolk sac endoderm

(A)、(B)calcium granules break up, (C)、(D)endocytosis to calcium granules

\section{Conclusion}

During development of incubation, the endoderm of yolk sac appeared large number of spherical crystal. There spherical crystal, growing from less to more and small to large, presented a growing trends. As the process of embryonic development, the wrinkle of endoderm increased gradually, which encased blood vessels. The endoderm full with spherical crystal, the spherical crystal assembled in the wrinkle of endoderm. SEM-EDS result and analysis comforted that the spherical crystal full with calcium. The substance of yolk sac transform from a disordered state to an ordered state (spherical crystal), promoting the absorption of calcium. The calcium absorption rate of endoderm gradually increases as the incubation time changes, which achieves maximum around middle stage of incubation, the calcium absorption rate declines slightly after incubation. The endoderm has the similar endocytosis to spherical calcium granules.

\section{Acknowledgment}

Financial support of this research was received from the National Natural Science Foundation of China (31071265) (to H.Tong)

\section{References}

[1] C.D. Holdsworth, T.H. Wilson, Development of active sugar and amino acid transport in the yolk sac and intestine of the chicken, Am. J. Physiol. 212(1967)233-240.

[2] S. G. Tullett, Fiona G. Burton, Factors affecting the weight and water status of the chick at hatch, J. Brit. Poultry Sci. 23 (1982)361-369. 
[3] Dr. Ian G. Mobbs, D. B. McMillan, Transport across endodermal cells of the chick yolk sac during early stages of development, Dev.Dynam.160 (1981)285-308.

[4] R.O. Lambson, An electron microscopic study of the entodermal cells of the yolk sac of the chick during incubation and after hatching. Dev.Dynam.129 (1970)1-19.

[5] M.F. Young, P. P. Minghetti, N.W. Klein, Yolk sac endoderm: Exclusive site of serum protein synthesis in the early chick embryo, Dev. Biol.75 (1980) 239-245.

[6] R. Bauer, J.A. Plieschnig, T.Finkes, B. Riegler, M. Hermann, W.J. Schneider, The developing chicken yolk sac acquires nutrient transport competence by an orchestrated differentiation process of its endodermal epithelial cells, J.Boil.Chem.288 (2013)1088-1098.

[7] R.S. Tuan, T.Ono, R.E.Akins, M.Koide, Experimental studies on cultured, shell-less fowl embryos: calcium transport, skeletal development, and cardio-vascular functions. In: Deeming DC, Ferguson MJW, editors. Egg Incubation: its effects on Embryonic Development in Birds and Reptiles. Cambridge: Cambridge University Press.1991. 419-433.

[8] A.R.Terepka, M.E. Stewart, N.Merkel, Transport functions of the chick chorioallantoic membrane. II. Active calcium transport, in vitro. Exp. Cell Res.58 (1969) 107-117.

[9] R. E.Dunn, J. S.Graves, T. P Fitzharris. Active calcium transport in the chick chorioallantoic membrane requires interaction with the shell membranes and/or shell calcium, Dev. Biol.88 (1981)259-268.

[10]N. B.Clark, M.Takada, Calcium transport across the yolk sac membrane of the chick embryo. Am.Zool,32 (1992)22-27.

[11]B. K.Speake, A. M.Murray, R. C. Noble, Transport and transformations of yolk lipids during development of the avian embryo, Prog. Lipid Res. 37 (1998)1-32.

[12]S.Komazaki, Ultrastructural localization of calcium in the chick yolk sac membrane endodermal cells as revealed by cytochemistry and X-ray microanalysis, Anat.Embryol. 187(1993)607-614.

[13]J.Song, H.X. Cheng, X.Y. Shen, J.X. Hu, H. Tong. Characterization of calcium carbonate crystals in pigeon yolk sacs with different incubation times, Micron 60(2014)39-48.

[14]J.Wakely, M.A. England, Development of the chick embryo endoderm studied by S.E.M..Anat.Embryol. 153(1978)167-178. 